\title{
Psychometric testing of the consequences of an HIV disclosure instrument in Mandarin: a cross- sectional study of persons living with HIV in Hunan, China
}

This article was published in the following Dove Press journal:

Patient Preference and Adherence

\author{
Xueling Xiao' \\ Junshi Zhao ${ }^{2}$ \\ Chulei Tang' \\ Xianhong $\mathrm{Li}^{\prime}$ \\ Jane M Simoni ${ }^{3}$ \\ Honghong Wang' \\ Kristopher P Fennie ${ }^{4}$
}

'Xiangya School of Nursing, Central South University, Changsha, People's Republic of China; ${ }^{2}$ Department of STD/AIDS, Center for Disease Control and Prevention of Hunan Province, Changsha, People's Republic of China; ${ }^{3}$ Department of Psychology, University of Washington, Seattle, WA, USA; ${ }^{4}$ Robert Stempel College of Public Health and Social Work, Florida International University, Miami, FL, USA
Correspondence: Honghong Wang Xiangya School of Nursing, Central South University, 172 Tong Zi Po Road, Changsha 4I00I3, Hunan, People's

Republic of China

$\mathrm{Tel}+8673$ I 82650270

Fax +86 73। 82650266

Email honghong_wang@hotmail.com
Purpose: This study aimed to examine the psychometric properties of a Mandarin-language version of an instrument that assesses the Consequences of HIV Disclosure (CoHD).

Patients and methods: The original CoHD instrument developed by Serovich was translated into Mandarin and administered to a random sample of 184 persons living with HIV (PLWH) using face-to-face and structured interviews. The CoHD instrument required respondents to rate the importance of eight costs (eg, might lose the relationship) and ten rewards (eg, would bring us closer) in their decision about whether to self-disclose their HIV status. The participants were directed to respond with respect to a current (or hypothetical) sexual partner.

Results: Internal consistency was acceptable (Cronbach's $\alpha$ for the overall scale 0.82 , costs 0.71 , and rewards 0.86 ), as was stability (test-retest reliability overall 0.74 , cost 0.63 , and rewards 0.82 ). The CVI for the scale was 0.83 , with items rated by subject experts ranging from 0.80 to 1.0. To determine structural validity, exploratory factor analysis extracted two subscales consistent with the original CoHD subscales. The Mandarin CoHD scores were significantly correlated with disclosure self-efficacy (indicating convergent validity), but they were unrelated to safer sex efficacy (indicating divergent validity). This criterion was tested by comparing the scores of PLWH who disclosed their HIV status (mean \pm SD 53.57 \pm 9.06 ) with those who did not disclose it (mean \pm SD 49.63 \pm 7.45 ); however, the difference was not statistically significant.

Conclusion: The Mandarin version of the CoHD instrument demonstrates promising psychometric properties when assessing costs and rewards with respect to sexual partner disclosure. This suggests that it might be useful in research on partner notification strategies. In further studies, larger and more diverse samples and an analysis of responses for different disclosure targets are warranted. Moreover, whether the CoHD score is related to the decision of disclosure should be determined.

Keywords: HIV, self-disclosure, sexual partner, partner notification, psychometric analysis, China

\section{Introduction}

In 2016, there were 36.7 million people worldwide who were living with HIV. ${ }^{1}$ From 2015 to 2016, 1.8 million people were diagnosed with HIV each year. ${ }^{2}$ In People's Republic of China, by the end of June 2016, the Chinese Center for Disease Control and Prevention (China CDC) reported 627,030 persons living with HIV (PLWH), and $>30,000$ newly infected cases were reported in the second quarter of 2016 . $^{3}$ Prevention strategies are urgently needed to stem the tide of HIV transmission. 
Partner notification (PN) is an effective strategy for HIV prevention. It involves PLWH being assisted in disclosing or being encouraged to disclose their seropositive status to sexual or drug-injecting partners if they agree to do so. ${ }^{4} \mathrm{PN}$ has been demonstrated to be effective in disease management. ${ }^{5}$ Moreover, incorporating into public health intervention strategies among PLWH can, potentially, have a positive impact on preventing the spread of this disease. If PLWH disclose their HIV-positive status to their sexual partners, they would be better able to take their antiretroviral drugs on time because they would have a simple explanation for taking these medications, and they would not have to worry that their partners know they are HIV positive. This helps to improve their medication adherence.${ }^{6,7}$ Furthermore, most sexual partners would accept that PLWH are HIV positive although the partners might have had some negative responses to that information when the PLWH told them about their status. At that point, the partners could prepare the PLWH to disclose their status to others. This would help PLWH to receive more support from their sexual partners and family members, accept HIV as part of their life, and perceive less of the stigma associated with the disease. ${ }^{8,9}$

Additionally, PN plays a critical role in enhancing the efficiency and coverage of HIV testing in PLWH's partners. PN is an approach that can be used to address the fact that $40 \%$ of an estimated 36 million PLWH are undiagnosed; moreover, PN could help to achieve the first goal of the United Nations' (UN's) 90-90-90 goal, which is to diagnose $90 \%$ of PLWH by $2020 .{ }^{4}$ Observational and experimental research studies have shown that the sexual partners of PLWH have an increased probability of being infected. ${ }^{10-13}$ The World Health Organization (WHO) reported that sexual partners of PLWH accounted for $18 \%$ of all new adult HIV infections globally in $2015 .{ }^{4} \mathrm{PN}$ has been positively related to a higher rate of HIV testing in sexual partners of PLWH, resulting in either new HIV diagnoses or the ability to prioritize effective protection so partners would not be infected. ${ }^{5,14}$ This has led to improvements in HIV testing among partners and the ability to diagnose more people. Moreover, it has led to a decline in the incidence of sexually transmitted infections.

PN is beneficial for both PLWH and public health, but its implementation has been challenging as PLWH might be reluctant to name their sexual partners ${ }^{15}$ or they might be afraid of revealing their serostatus to those partners. ${ }^{16,17}$ Intervention strategies are needed to promote PN. These strategies are significant because they will increase PLWH's understanding of the disclosure process and assess whether they are ready to tell their partners about their HIV-positive status. ${ }^{18}$ Therefore, psychometrically sound measurement tools that assess the decision-making process of HIV disclosure could be helpful for enhancing strategies, especially PN strategies, to bolster disclosure to sexual partners.

However, there are no Mandarin-language instruments to assess the disclosure process of PLWH. Most of the studies related to PN in People's Republic of China have evaluated whether PLWH had disclosed their HIV status to their partners based on individual self-reporting - namely, by asking PLWH whether they had disclosed their serostatus to their sexual partners - as well as by addressing associated factors; few studies have considered the cognitive process that is involved in PN. ${ }^{19-21}$

In 2000, Serovich ${ }^{22}$ developed an instrument to assess how PLWH rate the importance of various costs and rewards as they contemplate whether to reveal their HIV status. The Consequences of HIV Disclosure (CoHD) instrument, initially derived from the theory of consequences among men who have sex with men (MSM) ${ }^{22}$ consists of a list of eight costs and ten rewards. The aim of the CoHD is to ask PLWH to identify how important the list of possible consequences is to them after they reveal their HIV serostatus to a specific person, including their sexual partners. As indicated by the theory of consequence, if PLWH perceive more rewards and fewer costs, they would be more likely to disclose their HIV status. ${ }^{22,23}$ The original CoHD was first tested among 138 HIV-positive MSM. The Cronbach's $\alpha$ was 0.84 for costs and 0.79 for rewards. ${ }^{22}$ Another test was conducted among $125 \mathrm{HIV}$-positive women, and it was also found to have good psychometric features (Cronbach's $\alpha 0.86$ for costs and 0.87 for rewards) ${ }^{23}$ However, factor analysis has not yet been used to explore the factor structure of the CoHD.

Moreover, the CoHD instrument has not been translated into Mandarin, and it has not been applied in a cohort of Chinese PLWH. In comparison to other tools, CoHD was designed specifically to measure how important the consequences of disclosure to a specific person are in the PLWH's decision-making process. This instrument has good psychometrical features and it is easy to use. Therefore, the present study was designed to develop a Mandarin-language version of the CoHD instrument and psychometrically evaluate it.

\section{Patients and methods Setting and study team}

The research took place at the offices of the China CDC in the Hengyang and Yueyang districts of the Hunan Province in south central People's Republic of China from October 2014 to March 2015. The research team consisted of two nursing 
faculty from the Central South University who were trained in the United States (US), two Hunan CDC staff members, one epidemiology faculty from the International Florida International University, and four research assistants (two master's degree students, each, in nursing and public health).

\section{Instrument translation}

With approval from the original author of the CoHD scale, two investigators with strong English language skills who were also familiar with HIV infection and instrument development translated the CoHD scale into simplified Mandarin. Working with the research team, they engaged in discussions to reach consensus on a single Chinese version, which was back-translated into English by a bilingual professional who was trained in the US and familiar with HIV-related nursing. The back-translated English and original versions were compared, and final adjustments, consistent with cultural and linguistic considerations, were made. The adjusted Mandarin version of the CoHD was pilot-tested through structured and face-to-face interviews that were conducted by four research assistants. Twenty PLWH who met the inclusion and exclusion criteria were selected from two study sites. The research assistants read the questionnaire items, one by one, and asked the participants for their responses. The pilot test enabled the researchers to determine the appropriateness of the language and comprehensibility of the items. The final version of the Mandarin CoHD was achieved by modifying it on the basis of feedback received from the pilot test participants and discussions among members of the research team. The 20 PLWH who participated in the pilot test were excluded from the final study outcome.

\section{Participant recruitment}

In line with the standard convention that instrument-testing requires at least five to ten participants for each scale item, ${ }^{24}$ we aimed for a sample size of 200. Eligible participants were HIV-positive residents of Hengyang and Yueyang who were at least 18 years old and could speak Mandarin or a regional dialect. PLWH with cognitive impairment, active psychosis, or those who were receiving palliative care or participating in a different study were excluded. The China CDC staff created identification numbers for potentially eligible PLWH in Hengyang or Yueyang; the numbers were randomly sorted by a computer. A China CDC staff member telephonically contacted the first 200 individuals on the list and invited them to join the study. The final response rate was $92.0 \%(184 / 200)$. There were three reasons why 16 of the 200 PLWH did not participate in the study: they were not interested in the study $(n=8)$, moved to other places $(n=5)$, and lived too far away from where the survey was conducted $(n=3)$.

\section{Data collection}

Data were collected through structured and face-to-face interviews. Four trained research assistants conducted the data collection, and they asked the participants to response to the questionnaire items by reading the items, one by one and then marking the responses based on the participants' answers. In all, 184 PLWH participated in the final interview to test the psychometric features of the Mandarin CoHD; they provided written consent and participated in the interviews. Each interview lasted from 20 to 50 minutes. Each participant received 50 RMB (USD 7.50) as remuneration. The remaining $16 \mathrm{PLWH}$ (one refused) were invited to return 2 weeks later for a second interview to evaluate the test-retest reliability of the Mandarin CoHD.

\section{Measures}

The original CoHD is an 18-item instrument that asks respondents to rate how important (from 1 [not important] to 5 [very important]) is their decision to disclose their HIV status based on eight costs and ten rewards. The responses to costs are reverse recoded before summing all the items to produce a score for the consequences of disclosure, with higher scores reflecting an emphasis on rewards over costs. The original CoHD demonstrated good psychometric characteristics, and it has been tested among HIV-positive women and MSM. ${ }^{22,23}$

The Self-Efficacy Scale for Disclosing HIV Status and Safer Sex was developed by Kalichman et $\mathrm{al}^{25}$ to measure self-efficacy in the context of HIV disclosure to sexual partners and in negotiating safer sexual behaviors. It was included to facilitate the construct validity test for the present study. ${ }^{25,26}$ The instrument contains eight scenarios addressing two behavioral domains: disclosure and safer sex. Each of the subscales consists of five items scored on a four-point Likerttype scale (from 1 [strongly disagree] to 4 [strongly agree]). The internal consistency was excellent, with Cronbach's $\alpha$ ranging between 0.71 and $0.85 .^{25}$

Questions about the participants' sexual partners and their disclosure of their HIV status to those partners (if yes, who did the disclosure), were included in the general information sheet. Moreover, the basic sociodemographic characteristics of the participants were included.

\section{Data analysis}

To evaluate whether each item in the instrument should be included, the following criteria were used: 1) the percentage 
of participants choosing any one option of each item was $\geq 80 \%$; 2 ) the coefficient of variation (CV) was $<25 \%$; 3) $p$ was $\geq 0.05$ when comparing the critical ratio (CR) of each item using an independent-samples $t$-test; 4) the correlation of the item-total was $<0.3$; and 5) the correlation between one item and another subscale was $>0.4{ }^{27}$ If any item met any two of the criteria, it was removed from the instrument.

The factor structure of the translated CoHD was explored using exploratory factor analysis (EFA). Whether EFA could be conducted depended on the results of the Kaiser-MeyerOlkin (KMO) test of sample size and Bartlett's sphericity test. Principal component analysis was used to decide the final factors by evaluating the scree plot and the number of factors with eigenvalues $>1$.0. Items with factor loadings $\geq 0.4$ were considered to be meaningful. ${ }^{27}$

Internal consistency was assessed by Cronbach's $\alpha$; a value $\geq 0.7$ was acceptable. Fifteen participants were asked to complete the Mandarin CoHD 2 weeks later so that testretest reliability could be examined. Pearson's correlation coefficient was calculated; the resulting test-retest reliability value was $\geq 0.7$, indicating that the instrument had acceptable stability. ${ }^{27}$

The validity of the Mandarin CoHD was tested using the following methods: content validity, construct validity, and criterion reliability. Content validity was measured by five nursing, statistics, or HIV prevention experts. A four-point scale was used to assess the relevance of each item in the Mandarin CoHD to the underlying construct ( 1 = not relevant, $2=$ somewhat relevant, $3=$ relevant, and $4=$ highly relevant). The item content validity index (I-CVI) was computed as the number of experts assigning a rating of either three or four to an item divided by the total number of experts. The scale-CVI (S-CVI) was the average proportion of the Mandarin CoHD items that achieved a rating of three or four by the five content experts. An S-CVI $\geq 0.8$ was associated with acceptable content validity. ${ }^{28}$ Construct validity was evaluated by determining the correlation of the scale scores with the instruments, namely the subscales of the Self-Efficacy Scale for Disclosing HIV Status and Safer Sex that were conceptually similar (for convergent validity) or dissimilar (divergent validity). Criterion reliability was tested by comparing the Mandarin CoHD scores of PLWH who disclosed their HIV status with those who concealed it, as explained by the original author (in personal communication on October 19, 2017) who noted that the scale was designed with the expectation that higher scores would indicate that PLWH would be more likely to disclose their HIV status. Thus, the scores of PLWH who had revealed their serostatus would be significantly higher than those who had not. To analyze the relationships between demographical characteristics and the CoHD scores, an independent-samples $t$-test, Pearson's correlation, or analysis of variance (ANOVA) were used, as appropriate.

\section{Ethics statement}

This study was approved by the institutional review boards (IRBs) of Central South University and Florida International University. An verbal informed consent was acceptable and approved by the IRBs of two universities. The researchers explained the purpose of the study, the process of participation, and the risks/benefits of participation to the potential participants. The participants were told that they had the right to refuse to participate in the study, and their confidentiality was guaranteed. Either a written or verbal informed consent was obtained from all of the study participants.

\section{Results \\ Sociodemographic profile of the participants}

The final sample size was 184 . The participants ranged in age from 19 to 76; the mean age was 44.5 years (SD 10.30). Other demographics are summarized in Table 1.

\section{HIV disclosure status}

More than half $(104,56.5 \%)$ of PLWH had sex with partners after being diagnosed with HIV. According to the participants' self-report, 68 PWLH did self-disclose their HIV status to their sexual partners; 20 participants disclosed the status to their partners by having others relay the information, such as family members and the China CDC staff; and 16 participants did not disclose their HIV status to their sexual partners.

\section{Results of the items analysis}

Items analysis was used to evaluate the items and verify the homogeneity of the items in the preliminary Mandarin CoHD. The percentage of participants choosing any option of any item ranged from $1.1 \%$ to $57.6 \%$; thus, only $1.1 \%$ of the participants chose "very important" in response to the item "Thinking I could blow off steam to the person", whereas $57.6 \%$ selected "not important" in response to the item "Thinking we would fight if I told the person". The CR values of the 18 items ranged from 2.19 to 8.67. No items were removed from the Mandarin CoHD, as evidenced by the data shown in Table 2.

\section{EFA results for the structure of the Mandarin version of the CoHD}

The KMO value (0.82) and the sphericity results of Bartlett's test $\left(\chi^{2}=1,498.10, p=0.00\right)$ allowed us to conduct EFA to 
Table I Sociodemographic profile of the study participants

\begin{tabular}{|c|c|c|c|c|}
\hline \multirow[t]{2}{*}{ Variables } & \multirow[t]{2}{*}{ n (\%) } & \multicolumn{3}{|c|}{ Total CoHD scores and scores for the CoHD subscales } \\
\hline & & $\begin{array}{l}\text { Rewards } \\
(\text { mean } \pm \text { SD) }\end{array}$ & $\begin{array}{l}\text { Costs } \\
(\text { mean } \pm \text { SD) }\end{array}$ & $\begin{array}{l}\text { Total } \\
(\text { mean } \pm \text { SD })\end{array}$ \\
\hline \multicolumn{5}{|l|}{ Ethnicity } \\
\hline Han & I8I (98.4) & $24.9 \pm 8.53$ & $27.0 \pm 6.35$ & $51.9 \pm 9.36$ \\
\hline Others & $3(1.6)$ & $29.0 \pm 16.70$ & $25.7 \pm 3.79$ & $54.7 \pm 16.04$ \\
\hline \multicolumn{5}{|l|}{ Place of residence } \\
\hline Rural area & $63(34.2)$ & $24.3 \pm 7.55$ & $26.6 \pm 5.93$ & $50.9 \pm 8.46$ \\
\hline Others & $121(65.8)$ & $25.3 \pm 9.18$ & $27.2 \pm 6.51$ & $52.5 \pm 9.91$ \\
\hline \multicolumn{5}{|l|}{ Gender } \\
\hline Female & $5 \mathrm{I}(27.7)$ & $25.3 \pm 8.75$ & $25.9 \pm 7.21$ & $51.3 \pm 9.96$ \\
\hline Male & $133(72.3)$ & $24.9 \pm 8.64$ & $27.4 \pm 5.91$ & $52.3 \pm 9.26$ \\
\hline \multicolumn{5}{|l|}{ Education } \\
\hline Less than middle school & $22(12.0)$ & $21.9 \pm 7.20$ & $29.6 \pm 4.82$ & $51.5 \pm 7.10$ \\
\hline Middle school & $92(50.0)$ & $25.2 \pm 8.75$ & $27.0 \pm 6.26$ & $52.2 \pm 9.43$ \\
\hline High school & $43(23.4)$ & $25.1 \pm 8.14$ & $26.2 \pm 6.99$ & $51.3 \pm 10.19$ \\
\hline Technical training & $21(11.4)$ & $25.2 \pm 10.01$ & $26.4 \pm 6.46$ & $51.7 \pm 10.62$ \\
\hline College & $6(3.3)$ & $30.7 \pm 9.40$ & $26.3 \pm 5.68$ & $57.0 \pm 8.58$ \\
\hline \multicolumn{5}{|l|}{ Marital status } \\
\hline Never married & $44(23.9)$ & $29.3 \pm 10.30 *$ & $24.7 \pm 7.28 *$ & $54.0 \pm\left. 10.4\right|^{*}$ \\
\hline Married & $75(40.8)$ & $24.9 \pm 8.27$ & $27.5 \pm 5.63$ & $52.3 \pm 9.73$ \\
\hline Separated & $4(2.2)$ & $21.3 \pm 5.25$ & $25.3 \pm 5.85$ & $46.5 \pm 10.91$ \\
\hline Divorced & $45(24.5)$ & $23.4 \pm 5.86$ & $28.8 \pm 5.75$ & $52.1 \pm 6.78$ \\
\hline Widowed & $16(8.7)$ & $|9.2 \pm 7.9|$ & $26.7 \pm 6.81$ & $45.9 \pm 9.60$ \\
\hline \multicolumn{5}{|l|}{ Employed } \\
\hline Yes & $42(22.8)$ & $23.9 \pm 8.51$ & $27.7 \pm 5.71$ & $51.6 \pm 9.17$ \\
\hline No & $142(77.2)$ & $25.3 \pm 8.69$ & $26.8 \pm 6.48$ & $52.1 \pm 9.55$ \\
\hline \multicolumn{5}{|l|}{ Monthly income (Yuan) } \\
\hline No income & $5 \mathrm{I}(27.7)$ & $23.4 \pm 9.04$ & $26.5 \pm 6.33$ & $49.9 \pm 9.17^{\#}$ \\
\hline 500 or below & $65(35.3)$ & $26.3 \pm 8.09$ & $26.9 \pm 6.47$ & $53.1 \pm 9.36$ \\
\hline $50 I-1,000$ & $5(2.7)$ & $18.0 \pm 5.96$ & $27.0 \pm 5.00$ & $45.0 \pm 9.87$ \\
\hline $\mathrm{I}, 00 \mathrm{I}-\mathrm{I}, 500$ & $13(7.1)$ & $22.0 \pm 7.99$ & $26.2 \pm 7.30$ & $48.2 \pm 9.34$ \\
\hline $\mathrm{I}, 50 \mathrm{I}-2,000$ & $21(11.4)$ & $27.1 \pm 10.36$ & $29.7 \pm 7.05$ & $56.8 \pm 8.79$ \\
\hline $2,00 \mathrm{I}-2,500$ & $7(3.8)$ & $29.7 \pm 9.38$ & $24.1 \pm 5.82$ & $53.9 \pm 12.40$ \\
\hline $2,50 \mathrm{I}-3,000$ & $9(4.9)$ & $27.2 \pm 6.55$ & $27.8 \pm 4.82$ & $55.0 \pm 8.05$ \\
\hline 3,001 or above & $13(7.1)$ & $22.9 \pm 6.73$ & $27.2 \pm 4.49$ & $50.1 \pm 9.44$ \\
\hline
\end{tabular}

Notes: *Marital status was related to the scores for costs, rewards, and the total CoHD scale. \#Monthly income was related to the scores for the total CoHD scale. Abbreviation: CoHD, Consequences of HIV Disclosure.

evaluate the factor structure of the Mandarin version of the CoHD. The eigenvalues were found to be $>1$ for four of the factors. However, the scree plot showed that the slope line became flat for the third factor (Figure 1). Two factors for the Mandarin version of $\mathrm{CoHD}$, which accounted for $44.87 \%$ of the variance (Table 3 ), were finally extracted after principal component analysis and oblimin rotation for the sample data, suggesting that the factor structure of the Mandarin version of the CoHD was consistent with the original CoHD.

\section{Reliability of the Mandarin CoHD}

After testing the internal consistency of the instrument, Cronbach's $\alpha$ was found to be 0.82 for the both types of consequences; it was 0.71 for the costs consequences and
0.86 for the rewards consequences. Test-retest reliability was calculated using a subgroup $(n=15)$ of the sample. The value of Pearson's correlation coefficient was 0.74 for the overall scale, 0.63 for the subscale of costs, and 0.82 for the subscale of rewards.

\section{Validity of the Mandarin CoHD}

The I-CVI of the 18 items ranged from 0.80 to 1.00 , and the S-CVI was 0.83 . In terms of construct validity, the Pearson's correlation coefficient of scores of the Mandarin CoHD and the self-efficacy of disclosure was 0.15 (95\% CI 0.004-0.303, $p=0.04)$, indicating a modest convergent validity of the Mandarin CoHD. However, the Mandarin CoHD scores were not related to safer sex self-efficacy $(r=0.09$, $p=0.192$ ), and this demonstrated the divergent validity of 
Table 2 Items analysis of the Mandarin CoHD

\begin{tabular}{lllll}
\hline Items & $\begin{array}{l}\mathbf{C V} \\
(<\mathbf{2 5} \%)\end{array}$ & $\begin{array}{l}\boldsymbol{P} \text { for CR } \\
(\geq \mathbf{0 . 0 5})\end{array}$ & $\begin{array}{l}\text { Correlation } \\
\text { of item-total } \\
(<\mathbf{0 . 3})\end{array}$ & $\begin{array}{l}\text { Correlation between } \\
\text { item with another } \\
\text { subscale }(>\mathbf{0 . 4})\end{array}$ \\
\hline I* $^{*}$ & 0.45 & 0.00 & 0.36 & -0.03 \\
2 & 0.56 & 0.00 & 0.43 & -0.23 \\
$3^{*}$ & 0.31 & 0.03 & $0.20^{\mathrm{a}}$ & -0.19 \\
4 & 0.54 & 0.00 & 0.32 & -0.15 \\
5 & 0.52 & 0.00 & 0.54 & -0.20 \\
6 & 0.62 & 0.00 & 0.49 & -0.19 \\
$7^{*}$ & 0.51 & 0.00 & $0.30^{\mathrm{a}}$ & -0.08 \\
$8^{*}$ & 0.49 & 0.00 & 0.42 & -0.04 \\
$9^{*}$ & 0.44 & 0.00 & 0.42 & $-0.60^{\mathrm{b}}$ \\
10 & 0.34 & 0.00 & 0.35 & -0.16 \\
11 & 0.44 & 0.00 & 0.52 & -0.05 \\
12 & 0.56 & 0.00 & 0.57 & -0.14 \\
$13 *$ & 0.43 & 0.00 & 0.49 & -0.02 \\
$14 *$ & 0.44 & 0.00 & 0.53 & 0.01 \\
$15 *$ & 0.25 & 0.01 & $0.22^{\mathrm{a}}$ & -0.14 \\
16 & 0.61 & 0.00 & 0.58 & -0.16 \\
17 & 0.59 & 0.00 & 0.61 & -0.12 \\
18 & 0.61 & 0.00 & 0.63 & -0.15 \\
\hline
\end{tabular}

Notes: *The items belong to the costs consequences. ${ }^{2}$ The correlation of item-

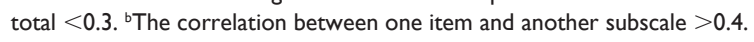

Abbreviations: CoHD, Consequences of HIV Disclosure; CV, coefficient of variation; $\mathrm{CR}$, critical ratio.

the Mandarin CoHD. In the present study, the Cronbach's $\alpha$ was 0.60 for self-efficacy of disclosure and 0.83 for selfefficacy of safer sex. Moreover, EFA showed that the structural consistency of the Mandarin version of the CoHD was similar to that of the original CoHD instrument. The scores of the cost consequences were negatively correlated to the scores of the rewards consequences $(r=-0.23, p=0.001)$. Therefore, the structure validity of the Mandarin version of CoHD was acceptable.

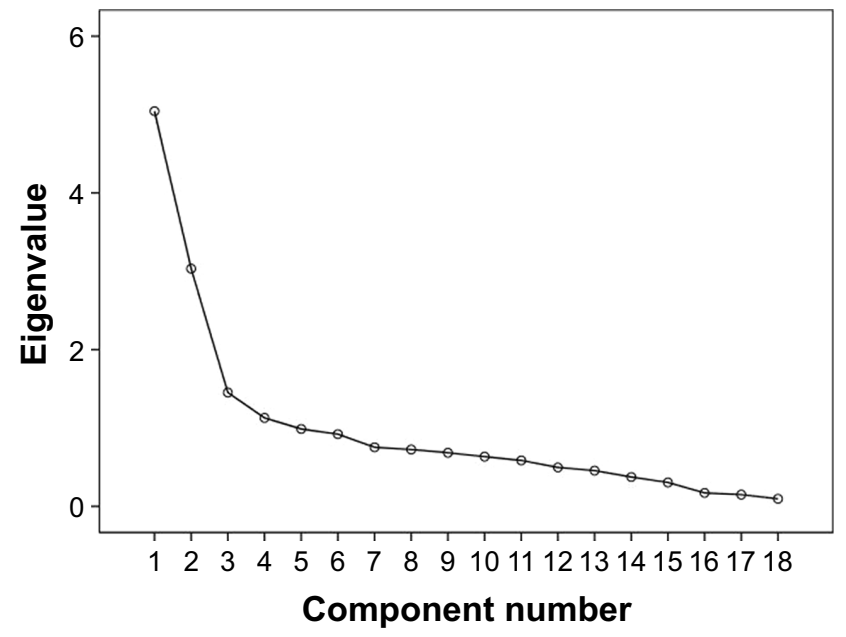

Figure I Scree plot.
Table 3 Factor loading of each item

\begin{tabular}{lll}
\hline Items & \begin{tabular}{l} 
Component \\
\cline { 2 - 3 }
\end{tabular} & $\begin{array}{l}\text { Factor I } \\
\text { (rewards) }\end{array}$ \\
\hline 18 & 0.83 & $\begin{array}{l}\text { Factor 2 } \\
\text { (costs) }\end{array}$ \\
16 & 0.80 & -0.07 \\
17 & 0.79 & -0.08 \\
5 & 0.72 & -0.04 \\
12 & 0.70 & -0.10 \\
6 & 0.69 & -0.05 \\
2 & 0.61 & -0.08 \\
11 & 0.54 & -0.17 \\
10 & 0.45 & 0.03 \\
4 & 0.43 & -0.11 \\
14 & 0.02 & -0.15 \\
13 & 0.00 & 0.85 \\
8 & -0.02 & 0.83 \\
9 & -0.02 & 0.69 \\
1 & -0.04 & 0.67 \\
15 & -0.15 & 0.56 \\
3 & -0.23 & 0.54 \\
7 & -0.07 & 0.54 \\
\hline
\end{tabular}

Criterion validity was examined by comparing the $\mathrm{CoHD}$ scores between PLWH who self-disclosed (mean 53.57, SD 9.06) to partners and those who did not disclose (mean 49.63, SD 7.45). Although a statistically significant difference was not found $(r=1.61, p=0.11)$, we still could see that PLWH who revealed their HIV status to partners had a higher CoHD score than those who did not.

\section{Mean score of the CoHD and related factors}

The scores of the CoHD ranged from 29 to 80, with a mean score of 52.0 (SD 9.44). For the rewards consequences, the mean was 25.0 (SD 8.65), with a range of 10 to 47 . For the costs consequences, the mean was 27.0 (SD 6.31). It was found that marital status and monthly income were related to the CoHD score, as shown in Table 1. Moreover, the age of subjects was related to the scores for costs $(r=0.18$, $p=0.016)$ and rewards $(r=0.26, p<0.001)$. The scores for each item are listed in Table 4.

\section{Discussion}

This study evaluated the psychometric properties of a Mandarin version of the CoHD instrument. Among a sample of 184 PLWH in south central People's Republic of China, we found the scale to have acceptable reliability and validity. All 18 items retained in the Mandarin CoHD were deemed to be comprehensible, discriminating, independent, and representative of the original CoHD items. 
Table 4 Scores of each item of the Mandarin CoHD*

\begin{tabular}{|c|c|c|c|}
\hline Costs & Mean \pm SD & Rewards & Mean \pm SD \\
\hline Thinking we would fight if I told the person & $4.28 \pm 1.08$ & Thinking I wanted the person to be safe from HIV infection & $3.83 \pm 1.30$ \\
\hline Thinking the person would lecture me & $3.95 \pm 1.22$ & Thinking the person had a right to know & $3.29 \pm 1.44$ \\
\hline Thinking I might lose the relationship with the person & $3.27 \pm 1.40$ & Thinking the person would be understanding & $2.77 \pm 1.43$ \\
\hline Thinking the person would have to be concerned about me & $3.22 \pm 1.41$ & Thinking I would get emotional support from the person & $2.61 \pm 1.46$ \\
\hline Thinking the person would blame me & $3.17 \pm 1.42$ & Thinking the person needed to be prepared to assist me & $2.46 \pm 1.39$ \\
\hline Thinking the person would not want to be around me & $3.16 \pm 1.54$ & Thinking I could count on the person & $2.24 \pm 1.40$ \\
\hline Thinking the relationship with the person would get bad & $3.15 \pm 1.38$ & $\begin{array}{l}\text { Thinking that telling the person would make our } \\
\text { relationship better }\end{array}$ & $2.02 \pm 1.24$ \\
\hline \multirow[t]{3}{*}{ Thinking the person would be burdened by the information } & $2.79 \pm 1.42$ & Thinking we would feel more connected & $2.01 \pm 1.19$ \\
\hline & & Thinking telling the person would bring us closer together & $1.96 \pm 1.19$ \\
\hline & & Thinking I could blow off steam to the person & $1.78 \pm 0.97$ \\
\hline
\end{tabular}

Note: *Costs and rewards items were obtained from Serovich and Serovich et al. ${ }^{22,23}$ Abbreviation: CoHD, Consequences of HIV Disclosure.

Specifically, the study's findings show that the reliability of the Mandarin version of CoHD is acceptable. Based on recommendations by both DeVellis and Nunnally as well as Bernstein that a Cronbach's $\alpha \geq 0.7$ indicates great internal consistency, ${ }^{29,30}$ the $\alpha$ value of 0.817 obtained for the whole instrument far surpassed the minimum standard thereby proving that the internal reliability of the Mandarin version is good. Moreover, it is worth mentioning that if the Cronbach's $\alpha$ is $>0.9$, the instrument might be redundant. ${ }^{31}$ Therefore, the Mandarin-language CoHD was found to have acceptable internal consistency and it was also easy to use. Moreover, the test-retest reliability was examined to evaluate the stability of the translated CoHD. A value of 0.74 for the total instrument exceeded the minimum value of 0.7 proposed by other researchers; ${ }^{32,33}$ this suggests that the repeated measures for the same attributes are consistent when using the Mandarin-language CoHD. Good internal reliability and stability contributed to the reliability of the Mandarin version of the CoHD. In another words, the translated instrument was reliable for measuring how PLWH would rate the importance of costs and rewards when deciding to disclose their HIV status.

Furthermore, the criterion validity of the Mandarin CoHD was demonstrated to be acceptable. EFA was used to explore the construct validity, and two factors emerged in accordance with the original CoHD, accounting for nearly half of the entire variance. All items loaded on the specific factor exceeded the criterion mentioned by Nunnally. ${ }^{30}$ Additionally, the divergent validity of the Mandarin CoHD was supported by the irrelevance of CoHD scores to safer sex efficacy. To some extent, convergent validity was also proven by the correlation between the CoHD scores and the disclosure self-efficacy, which was not very strong. The CoHD measures how PLWH think about the costs and rewards of disclosure when deciding on whether to disclose their HIV status. This is highly associated with their attitudes toward the possible or actual reactions they might encounter from their sexual partners. Positive attitudes toward rewards after disclosure could be affected by many factors. For example, it has been reported that PLWH that have a stronger interpersonal relationship with their sexual partners have a higher self-efficacy toward $\mathrm{PN},{ }^{34}$ indicating that self-efficacy is associated with positive rewards after PLWH reveal their serostatus. However, concerns about negative consequences after disclosure could make PN complicated, ${ }^{35}$ and this might negatively affect the correlation between self-efficacy and CoHD. CVI from five content experts was used to verify the content validity of the Mandarin version of the CoHD. ${ }^{36}$ According to Lynn's recommendation that a CVI $>0.78$ was good ${ }^{37}$ in the present study, the I-CVI ranged from 0.8 to 1.0 and the value of the S-CVI was 0.833 . This demonstrates that the content validity of the translated CoHD was strong. Thus, an adequate fit was demonstrated for assessing the perceived importance of consequences with the costs and rewards when PLWH consider disclosure.

However, criterion validity does not assume that PLWH with significantly higher scores would be more likely to disclose their HIV status. In the present study, we found that PLWH who had disclosed their HIV status to their sexual partners had a higher CoHD score. However, no statistically significant difference was found between the costs or rewards scores for PLWH who disclosed their status and those who did not. A potential reason for this outcome could be that our study had a small sample, especially in the non-disclosure group $(n=16)$. Another reason might be that the CoHD just considered the consequences of disclosure when used to measure the likelihood that PLWH would reveal or conceal their HIV status to their sexual partners. There is no doubt that 
the consequences of PN are an important factor, but future studies should consider more complex contributors, such as the sexuality, quality of life, and self-efficacy of PLWH. ${ }^{38,39}$ Furthermore, the wording of items might have contributed to the negative results. We suggest changing the wording of items, such as "How important is each of the following items in making decisions when considering telling someone about your HIV status?" to "How likely is each of the following items to occur if you consider disclosing your HIV status to a particular individual?" This would fit better with the theory of consequences; people who expected greater rewards and fewer costs would probably be more likely to disclose their HIV status, and vice versa. This is also indicated by other studies that suggested that the consequences of the costs of disclosure might impede PN. ${ }^{40-42}$ To say that a factor is "important" in one's decision to disclose is slightly different from saying one expected this to be a consequence of disclosure. For example, the consequence, "we get closer", might be an important for PLWH deciding on whether to disclose their status, but they might not expect to feel closer to their partner after making that disclosure in reality because their relationship was not close.

\section{Limitations}

It should be acknowledged that there were some limitations to this study. The first limitations are related to the size and diversity of the sample and the disclosure target. Because the subjects were only from the Hengyang and Yueyang districts of People's Republic of China, and the sexual partners of PLWH were the only target, it might be that the perceived importance of the consequences is not as common as it might be for other populations that are disclosing to family members or friends. Another limitation was that the criterion validity was not acceptable when taking the reality of disclosure as a criterion. However, the Mandarin CoHD was psychometrically appropriate for use as an instrument to measure the importance of the consequences that PLWH rate in regard to making decisions about disclosing their HIV status. Moreover, we did not add culturally relevant items to the Mandarin CoHD, which should be considered in the future based on more specific qualitative studies. However, the Mandarin CoHD could be used to effectively measure how PLWH think about the consequences of disclosure during their decision-making process, because their thoughts about the consequences could affect their decisions. ${ }^{38}$ In future psychometric testing, a larger and more diverse sample and different target populations in People's Republic of China are also warranted. Moreover, confirmatory factor analysis should be conducted to further validate and ensure the generalizability of the translated version of the CoHD.

\section{Conclusion}

The findings of this study showed that the Mandarin CoHD was reliable and had sufficient construct validity to assess how importantly PLWH in People's Republic of China would rate the costs and rewards of disclosing their HIV-positive status when contemplating sharing that information with their sexual partners in the real world. However, whether CoHD scores are related to real decisions about disclosing one's HIV status to one's sexual partners should be further determined. The CoHD instrument could provide a deeper understanding of the decision-making process of PN through quantified results, and it could allow public health staff to identify what PLWH value most in the process. This creates the opportunity to develop effective intervention strategies to potentially promote $\mathrm{PN}$ for PLWH based on their CoHD scores, and then provide specific services to PLWH and their referral partners.

\section{Acknowledgments}

The authors thank all the participants for participating in this study. This work was possible due to the support from the National Institute on Drug Abuse at the National Institutes of Health (grant no. R03DA025553). The authors appreciate the contributions from their colleagues, including Lu Zhou, Gangqiao Huang, Yan Jiang, and Zhisheng Liu.

\section{Author contributions}

$\mathrm{KP}$ Fennie and $\mathrm{HH}$ Wang were responsible for the design; XL Xiao, JS Zhao, and CL Tang conducted this study; XL Xiao and KP Fennie analyzed the data; XL Xiao, XH Li, JM Simoni, and $\mathrm{HH}$ Wang contributed to writing this paper. All authors contributed toward data analysis, drafting and revising the paper and agree to be accountable for all aspects of the work.

\section{Disclosure}

The authors report no conflicts of interest in this work.

\section{References}

1. Aidsinfo.unaids.org. UNAIDS: Number of people living with HIV. Available from: http://aidsinfo.unaids.org/. Accessed September 16, 2017

2. Aidsinfo.unaids.org. UNAIDS: Number of new HIV infections. Available from: http://aidsinfo.unaids.org/. Accessed September 16, 2017.

3. CDC C. Update on the AIDS/STD epidemic in China and main response in control and prevention the second quarter of 2016. Chin J AIDS STD. 2016;22(8):585.

4. WHO.int. WHO: Guidelines on HIV self-testing and partner notification: Supplement to consolidated guidelines on HIV testing services. Available from: http://www.who.int/hiv/pub/vct/hiv-self-testing-guidelines/en/. Accessed December 31, 2016.

5. Ferreira A, Young T, Mathews C, Zunza M, Low N. Strategies for partner notification for sexually transmitted infections, including HIV. Cochrane Database Syst Rev. 2013;(10):CD002843. 
6. Stirratt MJ, Remien RH, Smith A, Copeland OQ, Dolezal C, Krieger D; SMART Couples Study Team. The role of HIV serostatus disclosure in antiretroviral medication adherence. AIDS Behav. 2006;10(5): 483-493.

7. Kalichman SC, Kalichman MO, Cherry C, Grebler T. HIV disclosure and transmission risks to sex partners among HIV-positive men. AIDS Patient Care STDS. 2016;30(5):221-228.

8. Joge US, Deo DS, Choudhari SG, Malkar VR, Ughade HM. "Human immunodeficiency virus serostatus disclosure-rate, reactions, and discrimination": a cross-sectional study at a rural tertiary care hospital. Indian J Dermatol Venereol Leprol. 2013;79(1):135.

9. Maman S, van Rooyen H, Groves AK. HIV status disclosure to families for social support in South Africa (NIMH Project Accept/HPTN 043). AIDS Care. 2014;26(2):226-232.

10. Rosenberg NE, Mtande TK, Saidi F, et al. Recruiting male partners for couple HIV testing and counselling in Malawi's option B+ programme: an unblinded randomised controlled trial. Lancet HIV. 2015;2(11): e483-e491.

11. Brown LB, Miller WC, Kamanga G, et al. Predicting partner HIV testing and counseling following a partner notification intervention. AIDS Behav. 2012;16(5):1148-1155.

12. Xu Z, Wang Z, Li C. Analysis of HIV antibody test results in $232 \mathrm{HIV} /$ AIDS and their spouses or fixed sex partners. ChineseJ Health Lab Technol. 2013;23(5):1245-1247.

13. Hu L, Zhang Y, Li M, et al. Serostatus disclosure and associated factors among people living with HIV/AIDS (PLWHA) in Liuzhou city, China. Chin J Dis Control Prev. 2014;18(3):185-189. Chinese.

14. WHO.int. Consolidated guidelines on HIV testing services. Available from: http://www.who.int/hiv/pub/guidelines/hiv-testing-services/en/ Accessed May, 2016.

15. Adams OP, Carter AO, Redwood-Campbell L. Understanding attitudes, barriers and challenges in a small island nation to disease and partner notification for HIV and other sexually transmitted infections: a qualitative study. BMC Public Health. 2015;15(1):455-463.

16. Carnicer-Pont D, Barbera-Gracia MJ, Fernandez-Davila P, et al. Use of new technologies to notify possible contagion of sexually-transmitted infections among men. Gac Sanit. 2015;29(3):190-197.

17. Zhou L, Li Z, Wang H. Research progress on influencing factors of partner notification in HIV infection/AIDS patients. Chin Nurs Res. 2014;28(11):4106-4108.

18. WHO.int. WHO recommends assistance for people with HIV to notify their partners. Available from: http://apps.who.int/iris/ bitstream/10665/251548/1/WHO-HIV-2016.22-eng.pdf?ua=1 http:// www.who.int/hiv/pub/guidelines/hiv-testing-services/en/. Accessed December, 2016.

19. Chen J, Niu J, Wang Y. Status and influential factors of partner notification among people living with HIV/AIDS. China J Public Health. 2013;29(12):1742-1745

20. Tang X, Wu D, Wang S. A survey of HIV-positive patients' partner notification status in a city. World Latest Medicine Information. 2017; 17(7):35-36. Chinese.

21. Jin W, Zhong F, Xu H, Cheng W, Liu Q. Effects of notification via Easy Tell: an anonymous sexual partner notification system based on Internet and mobile phone. China J AIDS STD. 2016;22(5): 378-381.

22. Serovich JM. A test of two HIV disclosure theories. AIDS Educ Prev. 2001;13(4):355-364.
23. Serovich JM, Lim JY, Mason TL. A retest of two HIV disclosure theories: the women's story. Health Soc Work. 2008;33(1):23-31.

24. Tanaka JS. Sample size and goodness of fit in structural equation models with latent variables. Child Dev. 1987;58(1):134-146.

25. Kalichman SC, Rompa D, DiFonzo K, et al. Initial development of scales to assess self-efficacy for disclosing HIV status and negotiating safer sex in HIV-positive persons. AIDS Behavior. 2001;5(3):291-296.

26. Kalichman SC, Rompa D, Cage M, et al. Effectiveness of an intervention to reduce HIV transmission risks in HIV positive persons. Am J Prev Med. 2001;21(2):84-92.

27. Wu M. Statitical Analysis of Questionnaire: Operation and Using of SPSS. 1st ed. Chongqing: Chongqing University Press; 2015.

28. Yu H. The Preliminaiy Revision and Application of Maternal Breastfeeding Evaluation Scale. Changsha: Xiangya Nursing School, Central South University; 2012.

29. DeVellis RF. A Scale Development Theory and Application. 1st ed. London: SAGE; 1991.

30. Nunnally JC, Bernstein IH. Psychometric Theory. 3rd ed. New York: McGraw-Hill; 1994.

31. Streiner DL, Norman GR. Health Measurement Scales. A practical Guide to their Development and Use. 2nd ed. Oxford: Oxford University Press; 2000.

32. McCormack LA, Williams-Piehota PA, Bann CM, et al. Development and validation of an instrument to measure resources and support for chronic illness self-management: a model using diabetes. Diabetes Educ. 2008;34(4):707-718.

33. Terwee CB, Bot SD, de Boer MR, et al. Quality criteria were proposed for measurement properties of health status questionnaires. $J$ Clin Epidemiol. 2007;60(1):34-42.

34. Gursahaney PR, Jeong K, Dixon BW, Wiesenfeld HC. Partner notification of sexually transmitted diseases: practices and preferences. Sex Transm Dis. 2011;38(9):821-827.

35. Buchsbaum A, Gallo MF, Whiteman MK, et al. Sexually transmitted disease partner notification among African-American, adolescent women. Infect Dis Obstet Gynecol. 2014;2014:619632.

36. Davis LL. Instrument review: Getting the most from a panel of experts. Appl Nurs Res. 1992;5(4):194-197.

37. Lynn MR. Determination and quantification of content validity. Nurs Res. 1986;35(6):382-385.

38. Préau M, Beaulieu-Prévost D, Henry E, Bernier A, Veillette-Bourbeau L, Otis J. HIV serostatus disclosure: development and validation of indicators considering target and modality. Results from a communitybased research in 5 countries. Soc Sci Med. 2015;146:137-146.

39. Chaudoir SR, Fisher JD, Simoni JM. Understanding HIV disclosure: a review and application of the Disclosure Processes Model. Soc Sci Med. 2011;72(10):1618-1629.

40. Clark JL, Perez-Brumer A, Salazar X. "Manejar la Situacion”: partner notification, partner management, and conceptual frameworks for HIV/STI control among MSM in Peru. AIDS Behav. 2015;19(12):2245-2254.

41. Roberts J, Nixon E, Perry N, Sheriff N, Richardson D. Experiences of men who have sex with men (MSM) when engaging in the partner notification process three months following a HIV diagnosis. Sex Transm Infect. 2015;91(1):A36-A37.

42. Wayal S, Hart G, Copas A, Edwards S, Cassell JA. Sexual behaviour, partnership patterns and STI diagnoses among HIV positive MSM: implications for HIV/STIS transmission and partner notification. Sex Transm Infect. 2012;88(1):A7.
Patient Preference and Adherence

\section{Publish your work in this journal}

Patient Preference and Adherence is an international, peer-reviewed, open access journal that focuses on the growing importance of patient preference and adherence throughout the therapeutic continuum. Patient satisfaction, acceptability, quality of life, compliance, persistence and their role in developing new therapeutic modalities and compounds to optimize

\section{Dovepress}

clinical outcomes for existing disease states are major areas of interest for the journal. This journal has been accepted for indexing on PubMed Central. The manuscript management system is completely online and includes a very quick and fair peer-review system, which is all easy to use. Visit http://www. dovepress.com/testimonials.php to read real quotes from published authors. 